\title{
All the Wrong Places: An Unusual Case of Foreign Body Ingestion and Inhalation
}

\author{
V.S. Tammana N. Valluru A. Sanderson \\ Howard University Hospital, Washington, D.C., USA
}

\section{Key Words}

Foreign bodies in the stomach $\cdot$ Bronchus $\cdot$ Colon

\begin{abstract}
Intentional ingestion of foreign bodies is common in psychiatric patients and prison inmates. Timing of endoscopy for ingested foreign bodies varies and depends on the type and location of the foreign body in the gastrointestinal tract. We present the case of a 26-year-old man who was brought from a correctional facility after confessing to have swallowed a few shower curtain hooks. Abdominal X-ray done in the emergency room revealed multiple foreign bodies in the stomach. An upper endoscopy was done in the emergency room with the use of an overtube. The first metal piece was caught by a snare and removed with the endoscope. All other foreign bodies which were present on the abdominal X-ray could not be visualized initially as there was retained food in the stomach. After multiple attempts, four other foreign bodies were found and each one was caught by the forceps and then the scope was removed with the forceps holding the foreign body. There was an additional foreign body in the right mainstem bronchus. The patient had coughed up the foreign body and swallowed it into the gastrointestinal tract. A computed tomography scan of chest and abdomen was done for evaluation, which showed the foreign body in the cecum. To our knowledge, this is the first case report of a patient intentionally transferring a foreign body from one organ system to another. Colonoscopy was done and the foreign body was removed rectally with a snare without any complications.
\end{abstract}

\section{Introduction}

Voluntary or involuntary ingestion of foreign bodies can occur in adult or pediatric patients. Intentional ingestion of foreign bodies is common in psychiatric patients and prison inmates $[1,2]$.Various types of foreign bodies ingested are toothbrushes, pens, plastic spoons, pencils, batteries, food bolus, paper clips, razor blades, coins, bones, pins and pills [1-3]. Foreign bodies in children are usually coins [3]. Once in the 
stomach, most ingested foreign bodies pass without any intervention [3]. Foreign bodies can get lodged in the esophagus, stomach, small bowel or rectum. Generally, this occurs in narrow areas (i.e. pylorus and ileocecal valve). The inability to swallow saliva, dysphagia and neck tenderness are common clinical features in foreign bodies of the esophagus. Abdominal pain, bowel obstruction or perforation can occur with sharp objects in the esophagus, stomach, small bowel or colon. Few recent studies have shown that in the setting of intentional ingestion, the rate of endoscopic intervention may be $63-76 \%$ and the need for surgical intervention ranges from 12 to $16 \%$ [1, 4]. In a study of predominantly intentional ingestions, a success rate of $90 \%$ with endoscopic extraction was observed [1]. All sharp and pointed foreign bodies should be removed before they pass from the stomach because of the risk of intestinal perforation [5]. Timing of endoscopy for ingested foreign bodies varies and depend on patient age, clinical condition, type, size, shape, content and anatomic location of the foreign body in the gastrointestinal tract (table 1) [6]. Rat tooth forceps and snares are the most commonly used accessory devices to remove foreign bodies [7]. Disk batteries are retrieved with a net or basket successfully [8]. An overtube can be used while removing the foreign body to prevent accidental slippage of the foreign body into the trachea $[1,9]$. Drug packets have a risk of rupture and leakage of contents can be fatal, so endoscopic removal should not be attempted [6]. Rectal foreign bodies can be removed transanally. We present a case of intentional ingestion and inhalation of shower curtain hooks.

\section{Case Report}

A 26-year-old man with a past medical history of bipolar disorder, schizophrenia and diabetes mellitus was brought from a correctional facility after confessing to have swallowed a few shower curtain hooks. The patient heard some inner voices telling him to kill himself. He then straightened a few shower curtain hooks and swallowed them. He complained of chest pain, shortness of breath and abdominal pain with nausea and vomiting. His review of systems was positive for productive cough and fever. He had a history of pacemaker placement for unclear reasons. Outpatient medications included metformin, metoprolol, sublingual nitroglycerin, Percocet, Baby Aspirin, Risperdal and trazodone. He had no known drug allergies. He was a 10 pack-year smoker and denied alcohol and drugs. Family history is not significant. Abdominal X-ray done in the emergency room revealed multiple foreign bodies in the stomach (fig. 1a, yellow arrow). Labs revealed normal metabolic panel, hemoglobin $14 \mathrm{~g} / \mathrm{dl}$, white blood cells $8,100 / \mathrm{mm}^{3}$, platelets $213,000 / \mathrm{mm}^{3}$, mean corpuscular volume $85 \mathrm{fl}$ and international normalized ratio 1.01 .

An upper endoscopy was done in emergency room, with the use of an overtube, which showed the metal pieces as well as retained food in the stomach and gastritis. The first metal piece was caught by a snare and removed with the endoscope. All other foreign bodies which were present on the abdominal X-ray could not be visualized initially as there was retained food in the stomach. Then a rat tooth forceps was passed through the accessory channel and fishing for foreign bodies was attempted in the retained food material. After multiple attempts, four other foreign bodies were found and each one was caught by the forceps and then the scope was removed with the forceps holding the foreign body. Though three were removed easily, the last foreign body kept slipping back into the esophagus when the endoscope was pulled into the overtube. So finally, when the last foreign body, which was held by forceps, was pulled into the overtube it was removed in total with the endoscope and the rat tooth forceps holding the foreign body. The extracted metal pieces were about $7 \mathrm{~cm}$ in length ( $\underline{\mathrm{fig} .}$ ). Upon review of the chest X-ray, the result of which was not available at the time of endoscopy, there was an additional foreign body in the right mainstem bronchus (fig. 1b, yellow arrow). Pulmonary and subsequently cardiothoracic surgery were consulted for a rigid bronchoscopy and possible retrieval of the foreign body. On hospital day 1 , a repeat chest X-ray was done, which failed to show the foreign body which was originally present in the right mainstem bronchus. A computed tomography (CT) 
scan of the chest and abdomen was done for evaluation, which showed the foreign body in the cecum. The patient had coughed up the foreign body and swallowed it into the gastrointestinal tract. The patient was monitored for 3 days with serial abdominal X-rays (fig. 1c and d, yellow arrows), but the foreign body did not pass. He was ultimately given goLYTELY for bowel preparation and a colonoscopy was planned to remove the foreign body. Colonoscopy was done and the foreign body was caught by the snare with the long axis of the foreign body oriented in parallel to the long axis of the colon, and the colonoscope with the snare holding the foreign body was pulled out slowly and safely from the patient's rectum without any complications.

\section{Discussion}

Ingestion of foreign bodies can occur in adults or children. Patients with foreign body ingestion should be evaluated for obstruction or perforation. This case report describes the rare scenario of a patient ingesting six shower curtain hooks of which five were found in the stomach and one in the right mainstem bronchus. The presence of food material in the stomach can pose a challenge for the endoscopist trying to remove the foreign body safely. Radiologic studies such as X-rays or CT scans are helpful in localization of the foreign body in the gastrointestinal tract, in assessing the shape and size of the foreign body and in planning management appropriately, if retrieval is warranted. Illegal drugs wrapped in packets can usually be seen radiographically, and CT scanning may be helpful, although false-negative scan results have been reported [10]. Blunt objects in the stomach pass spontaneously within 4-6 days. With sharp objects in the stomach, complications can occur in up to $15-35 \%$ of cases $[5,11]$ and hence every attempt has to be made to remove them endoscopically. Long objects $(6-10 \mathrm{~cm})$ are unlikely to pass into the duodenal sweep and should be removed [1]. We used a snare and a rat tooth forceps to remove the foreign bodies. We also used an overtube at the time of endoscopy to remove them. A hood can also be used while removing the foreign body to prevent accidental slippage [1]. Once the foreign body is pulled into the overtube with an endoscope, all three can be removed together from the patient's mouth in one single motion to avoid losing grasp of the object within the overtube [12]. We used this technique in removing the last foreign body from the stomach.

Upon careful review of the initial abdominal X-ray a small tip of the foreign body in the bronchus can be viewed (fig. 1a, blue arrow). This was mistakenly thought to be part of the patient's pacemaker apparatus. The presence of an additional foreign body in the right mainstem bronchus prompted surgical consultation for rigid bronchoscopy, but before the patient was taken to the operating room, he coughed up the foreign body and swallowed it. To our knowledge, this is the first case report of a patient intentionally transferring a foreign body from one organ system to another. The foreign body was later identified in the cecum on abdominal CT. A foreign body which has crossed the ileocecal valve can spontaneously pass per rectum. Therefore, the patient was observed to see whether the object was progressing to the distal rectum. As the foreign body was in the colon for 3 days without any rectal passage, endoscopic retrieval was performed to shorten the waiting time to natural expulsion, given the patient was from a correctional facility and might have re-ingested the foreign body if left to his own devices. 
In conclusion, the current case illustrates the unusual probability of simultaneous foreign bodies in the airway and gastrointestinal tract and the success of endoscopy in safely retrieving the foreign bodies from the stomach and colon.

Table 1. Timing of endoscopy for ingested foreign bodies

\section{Emergent endoscopy}

Patients with esophageal obstruction (i.e. unable to manage secretions)

Disk batteries in the esophagus

Sharp-pointed objects in the esophagus

Urgent endoscopy

Esophageal foreign objects that are not sharp-pointed

Esophageal food impaction in patients without complete obstruction

Sharp-pointed objects in the stomach or duodenum

Objects $>6 \mathrm{~cm}$ in length at or above the proximal duodenum

Magnets within endoscopic reach

Non-urgent endoscopy

Coins in the esophagus may be observed for $12-24 \mathrm{~h}$ before endoscopic removal in asymptomatic patients

Objects in the stomach with a diameter $>2.5 \mathrm{~cm}$

Disk batteries and cylindrical batteries that are in the stomach of patients without signs of gastrointestinal injury may be observed for as long as $48 \mathrm{~h}$; batteries remaining in the stomach longer than $48 \mathrm{~h}$ should be removed 


\begin{tabular}{r|l|l|l}
$\begin{array}{r}\text { Case Reports in } \\
\text { Gastroenterology }\end{array}$ & $\begin{array}{l}\text { Case Rep Gastroenterol 2012;6:778-783 } \\
\text { DOl: 10.1159/000346287 }\end{array}$ & $\begin{array}{l}\text { Published online: } \\
\text { December 20, 2012 }\end{array}$ & $\begin{array}{l}\text { @ 2012 S. Karger AG, Basel } \\
\text { ISSN 1662-0631 } \\
\text { www.karger.com/crg }\end{array}$ \\
\hline
\end{tabular}
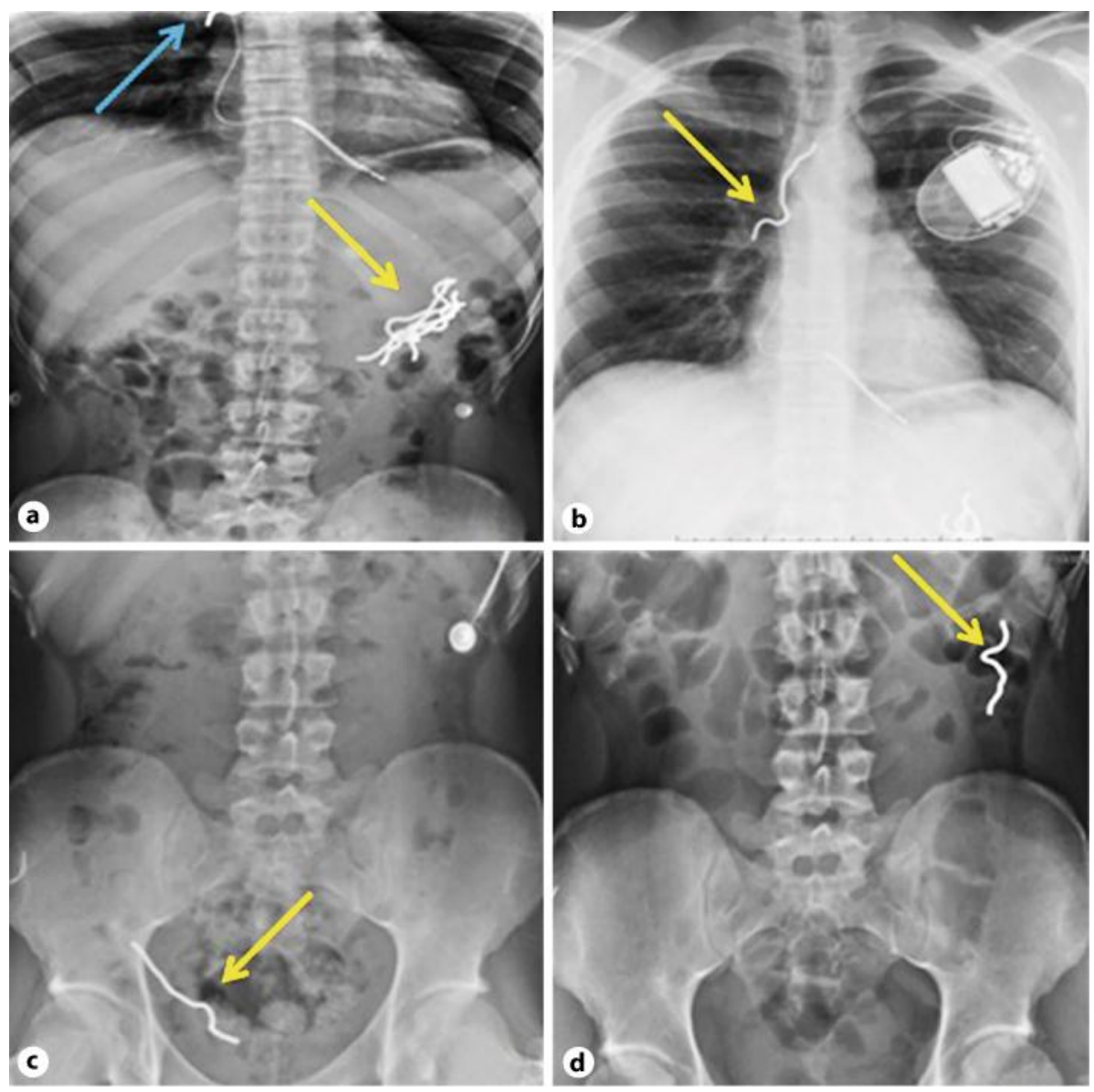

Fig. 1. a Foreign body in the stomach (yellow arrow) and bronchus (blue arrow). b Foreign body in the right mainstem bronchus (yellow arrow). c Foreign body in the right colon (yellow arrow). d Foreign body in the left colon (yellow arrow).

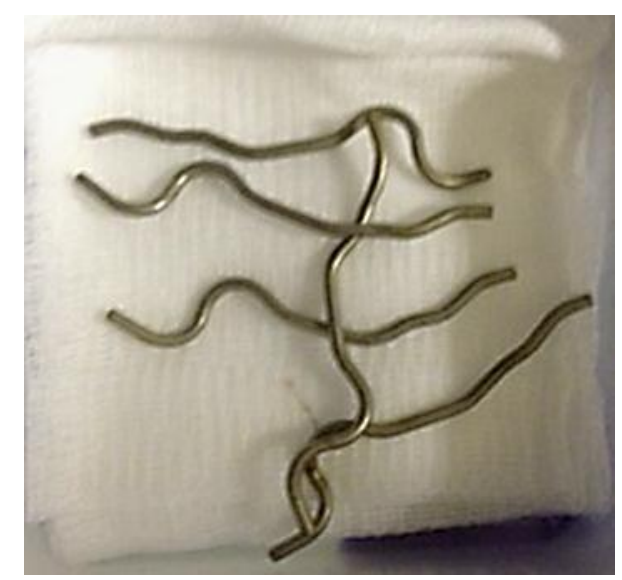

Fig. 2. Endoscopically removed foreign bodies. 


\section{References}

$>1$ Palta R, Sahota A, Bemarki A, Salama P, Simpson N, Laine L: Foreign-body ingestion: characteristics and outcomes in a lower socioeconomic population with predominantly intentional ingestion. Gastrointest Endosc 2009;69:426-433.

$\checkmark 2$ Blaho KE, Merigian KS, Winbery SL, et al: Foreign body ingestions in the emergency department: case reports and review of treatment. J Emerg Med 1998;16:21-26.

-3 Webb WA: Management of foreign bodies of the upper gastrointestinal tract: update. Gastrointest Endosc 1995;41:39-51.

-4 Weiland ST, Schurr MJ: Conservative management of ingested foreign bodies. J Gastrointest Surg 2002;6:496-500.

5 Rosch W, Classen M: Fiberendoscopic foreign body removal from the upper gastrointestinal tract. Endoscopy 1972;4:193-197.

6 ASGE Standards of Practice Committee: Management of ingested foreign bodies and food impactions. Gastrointest Endosc 2011;73:1085-1089.

$>7$ Li ZS, Sun ZX, Zou DW, et al: Endoscopic management of foreign bodies in the upper GI tract: experience with 1,088 cases in China. Gastrointest Endosc 2006;64:485-492.

8 Faigel DO, Stotland BR, Kochman ML, Hoops T, Judge T, Kroser J, Lewis J, Long WB, Metz DC, O’Brien C, Smith DB, Ginsberg GG: Device choice and experience level in endoscopic foreign object retrieval: an in vivo study. Gastrointest Endosc 1997;45:490-492.

>9 ASGE Technology Committee, Tierney WM, Adler DG, Conway JD, et al: Overtube use in gastrointestinal endoscopy. Gastrointest Endosc 2009;70:828-834.

10 Eng JGH, Aks SE, Marcus C, et al: False-negative abdominal CT scan in a cocaine body stuffer. Am J Emerg Med 1999;17:702-704.

11 Carp L: Foreign bodies in the intestine. Ann Surg 1927;85:575-591.

12 Chinitz MA, Bertrand CZ: Endoscopic removal of toothbrushes. Gastrointest Endosc 1990;36:527-530. 